\title{
DESENVOLVIMENTO DE UM VANT AUTÔNOMO UTILIZANDO HARDWARE E MATERIAIS DE BAIXO CUSTO
}

\section{DEVELOPMENT OF AN AUTONOMOUS VANT USING LOW COST HARDWARE AND MATERIALS}

Andre Mescoloti; Francisco Assis da Silva; Leandro Luiz de Almeida; Danillo Roberto Pereira; Mario Augusto Pazoti

${ }^{1}$ Universidade do Oeste Paulista - UNOESTE, Faculdade de Informática - FIPP, Presidente Prudente, SP.

E-mail: chico@unoeste.br

RESUMO - Os VANTs (Veículos Aéreos não Tripulados) são muito utilizados para realizar muitas atividades em diversas áreas. Apesar da grande evolução da tecnologia e o surgimento de modelos e marcas no mercado, a aquisição de um desses modelos comerciais ainda é bem custosa. Busca-se com este trabalho integrar tecnologias e materiais de baixo custo para propor uma solução mais viável. O trabalho tem como objetivo desenvolver um VANT autônomo de custo reduzido para realizar voos controlados seguindo uma rota estipulada. A rota é um percurso planejado (plano de voo) na estação de controle (computador em solo) cujo desenvolvimento também faz parte deste trabalho. Foi escolhido o modelo de quadrirrotor para a construção do VANT idealizado, que é uma espécie de helicóptero que possui uma estrutura em forma de "X", constituído de 4 motores acoplados com hélices em cada umas das extremidades.

Palavras-chave: VANT; Quadrirrotor; Arduino; ZigBee; GPS.

Recebido em: 14/04/2017

Revisado em: 10/09/2017

Aprovado em: 20/10/2017
ABSTRACT - The UAVs are widely used to carry out many activities in various areas. Despite the great evolution of technology and the emergence of models and brands in the market, the acquisition of one of these commercial models is still very expensive. This work seeks to integrate technologies and materials of low cost to propose a more feasible solution. The work aims to develop a self-contained low-cost VANT to conduct controlled flights following a stipulated route. The route is a planned course (flight plan) at the control station (ground computer) whose development is also part of this work. The quadrirrotor model was chosen for the construction of the idealized VANT, which is a kind of helicopter that has an "X" shaped structure, consisting of 4 motors coupled with propellers at each end.

Keywords: VANT; Quadrirrotor; Arduino; ZigBee; GPS. 


\section{INTRODUÇÃO}

Um veículo aéreo não tripulado (VANT, em inglês Unmanned Aerial Vehicle, UAV) é um tipo de aeronave que não necessita de um piloto embarcado para pilotá-la. Seu modo de operação pode ser feito de maneira remota ou autônoma ou uma combinação de ambas (US DEPARTMENT OF DEFENSE, 2010). SUa utilização geralmente consiste em decolar, seguir rota até $O$ seu destino, coletar informações e retornar a origem (PAULA, 2012). Os UAV são aeronaves não tripuladas, desenvolvidas por equipes militares dos Estados Unidos da América, pois permitiam a observação do campo dos inimigos sem riscos à vida humana caso a aeronave fosse atingida (FOWERS, 2008).

Com o avanço tecnológico nos setores de processamento de dados e miniaturização de componentes eletrônicos ocorridos nas últimas décadas, diversas aplicações militares de VANTs foram desenvolvidas ao redor do mundo, havendo constante crescimento do número de projetos (ARMADA, 2004). Mais recentemente, usos científicos e civis têm sido desenvolvidos, nos quais veículos aéreos sem tripulação podem apresentar vantagens técnicas e econômicas em relação a diferentes campos de atuação. Na maioria das aplicações desenvolvidas, os VANTs têm sido concebidos como plataformas para embarcar sensores remotos para obtenção de imagens e dados da superfície terrestre com o uso de sensores. O sensoriamento remoto por VANTs apresenta vantagens em relação ao aerotransportado por aeronaves tripuladas e ao orbital, tais como: (i) redução dos custos de obtenção de imagens/fotografias aéreas; (ii) maior flexibilidade para obtenção de imagens de alta resolução espacial; (iii) possibilidade de execução de missões em condições adversas sem risco de vida para o piloto e operador da câmera aerofotogramétrica; (iv) menores gastos de treinamento de pilotos e; (v) maior facilidade de incorporação de novas tecnologias (LONGHITANO, 2010).
Paula (2012) também destaca as principais vantagens no uso de VANTs frente às aeronaves tripuladas, em que o custo de produção e manutenção são menores, e possui maior flexibilidade em locais de difícil acesso e longas jornadas de voo.

Após anos de pesquisa neste campo, foi criada uma diversidade de aeronaves capazes de voar sem um piloto fisicamente presente na aeronave. As aeronaves criadas foram categorizadas cada uma de acordo com as suas características em voo. As aeronaves de asa fixa, mais conhecidas como aviões, eram movidas por motores com hélices, ou por motores a jato. Devido às suas limitações, as imagens capturadas por este tipo de aeronave não são nítidas, devido a dificuldades existentes para se pairar no mesmo ponto referencial. Deste modo, era necessário sobrevoar regiões em grandes altitudes de modo a conseguir circundar a região alvo. Portanto, tais fatores levaram a criação dos VTOL (Vertical TakeOff and Landing). Os VTOL permitem um voo em uma altitude mais baixa, consequentemente melhores qualidades de imagens. Além disso, podem decolar e pousar em qualquer superfície sem a necessidade de pistas de decolagem. $O$ desenvolvimento deste tipo de equipamento ocasionou uma redução considerável nos tamanhos das aeronaves, reduzindo assim os custos de construção e aumento de sua popularidade. Passou de motores movidos a combustão para motores elétricos, e devido a ao fato de serem menores e leves, começaram a ser utilizados em voos em espaços fechados, ao invés de apenas ao ar livre e céu aberto. Ao longo do tempo, a estrutura dos VTOL foi melhorada, como forma de aprimorar seu desempenho em voo e conseguir suportar carga, com o objetivo de transportar sensores para as mais variadas análises e missões. Assim, os VTOL evoluíram do helicóptero tradicional para uma estrutura mais estável: os multicópteros, que possuem desde 4 a 8 motores equidistantes do centro, proporcionando um poder maior de 
elevação, assim como um voo mais estável (FOWERS, 2008; EISENBEI, 2009).

Pela complexidade e os altos custos envolvidos, essa foi uma tecnologia que se manteve restrita para uso militar por várias décadas. Os VANTs comerciais começaram a surgir na década de 80 e como a tecnologia passou a ser cada vez mais acessível, eles vem sendo utilizados largamente nas mais diversas aplicações civis, como demonstrado na Tabela 1 (WONG, 1997).

Tabela 1. Algumas aplicações civis onde os VANTs estão sendo aplicados.

Fonte: (WONG, 1997).

\begin{tabular}{|c|c|}
\hline Indústria & Uso \\
\hline Indústria agrícola & $\begin{array}{lr}\text { Fotografia aérea } & \text { para } \\
\text { monitoramento } & \text { de } \\
\text { crescimento e } & \text { praga } \\
\text { nas plantações } & \\
\text { Aplicação } & \text { de } \\
\text { fertilizantes } & \text { e } \\
\text { pesticidas. } & \end{array}$ \\
\hline Pesquisas climáticas & $\begin{array}{l}\text { Coleta de dados } \\
\text { atmosféricos. }\end{array}$ \\
\hline Exploração de minérios & $\begin{array}{l}\text { Reconhecimento aéreo } \\
\text { para encontrar } \\
\text { minerais em áreas de } \\
\text { difícil acesso. }\end{array}$ \\
\hline $\begin{array}{l}\text { Guarda costeira e } \\
\text { policiamento urbano }\end{array}$ & $\begin{array}{l}\text { Monitoramento aéreo } \\
\text { de fronteiras, costas e } \\
\text { vias públicas. }\end{array}$ \\
\hline Telecomunicações & $\begin{array}{l}\text { Plataforma } \\
\text { retransmissora de } \\
\text { sinais. }\end{array}$ \\
\hline Energia & $\begin{array}{l}\text { Monitoramento de } \\
\text { linhas de transmissão. }\end{array}$ \\
\hline Televisão e cinema & Tomadas aéreas. \\
\hline Entretenimento & $\begin{array}{l}\text { Brinquedo, } \\
\text { aeromodelismo, etc. }\end{array}$ \\
\hline
\end{tabular}

O conceito do quadrirrotor já é antigo, surgiu no inicio do século XX onde em 1907 foi criado o primeiro quadrirrotor que se tem registro, ele foi criado por Charles Richet e os irmãos Bréguet (Figura 1), chamado de "Bréguet-Richet Quad-Rotor Helicopter". Um modelo que necessitava de um piloto embarcado e seu sistema de controle era manual através de mecanismos mecânicos simples, o quadirrotor pesava cerca de $578 \mathrm{~kg}$. Segundo relatado o protótipo não conseguiu obter a estabilidade necessária para sua pilotagem, não levantando mais que um metro e meio do solo (LEISHMAN, 2002).
Figura 1. Primeiro quadrirrotor, BréguetRichet Quad-Rotor Helicopter.

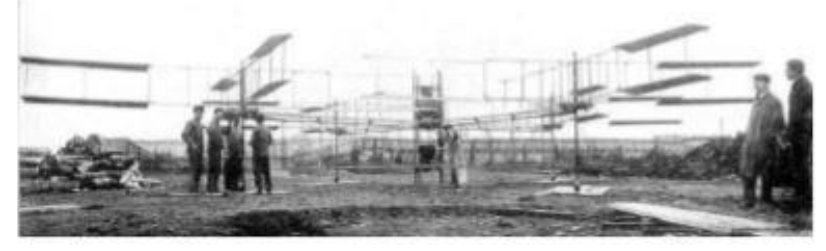

Fonte: (LEISHMAN, 2002).

Por prover de um sistema mecânico simples e barato, o quadrirrotor vem sendo utilizado atualmente nas mais diversas áreas comerciais e acadêmicas, como as indústrias de entretenimento, reconhecimento aéreo, tomada de imagens, etc. Este tipo de aeronave é utilizado no meio acadêmico 
como objeto de estudo de novas estruturas físicas, sensores, estratégias de controle, etc. (PAULA, 2012).

Apesar de não conseguir um funcionamento satisfatório, Charles Richet e os irmãos Bréguet contribuíram descrevendo a teoria dos pares de hélices girarem em sentidos opostos, conceito ainda hoje utilizado para evitar o torque giroscópio (LEISHMAN, 2002).

Neste trabalho foi realizada a construção de um VANT para voos autônomos, buscando integrar sensores de GPS (Global Positioning System), barômetro, magnetômetro e acelerômetro e giroscópio para estabilidade. Para a realização de voos controlados (plano de voos), foi desenvolvida uma controladora embarcada no VANT utilizando a placa de prototipagem Arduino ${ }^{1}$ juntamente com os sensores integrados. Foi desenvolvida uma aplicação (Estação de Controle) para ser executada no Sistema Operacional Windows utilizando recursos de mapas para planejamento de rotas e obtenção das informações do VANT em voo por meio da telemetria. A telemetria foi realizada utilizando a comunicação de dados do shield XBee.

As demais seções deste trabalho estão organizadas da seguinte maneira: na Seção 2 são apresentados os trabalhos correlatos, que serviram para inspirar a realização deste; na Seção 3 é detalhada a metodologia proposta; na Seção 4 são apresentados os experimentos realizados; por fim, a Seção 5 traz as considerações finais e propostas para trabalhos futuros.

\section{TRABALHOS RELACIONADOS}

Bresciani (2008) desenvolveu um quadrirrotor (Figura 2) utilizando uma estrutura construída de tubos de carbono. Para o controle do quadrirrotor foi utilizado um sistema eletrônico processado por um microcontrolador ARM7-STR730. Para os

\footnotetext{
1 Arduino: é uma plataforma de prototipagem eletrônica de hardware livre e de placa única, projetada com um microcontrolador Atmel AVR com suporte de entrada/saída embutido, geralmente programado na linguagem de programação $\mathrm{C} / \mathrm{C}++$.
}

sensores inerciais foi utilizado uma unidade de medidas inerciais (IMU - Inertial Mensurament Unit) modelo MTi-G composta de acelerômetro, giroscópio, magnetômetro e GPS. Segundo o autor, os testes realizados foram satisfatórios no voo pairado com uma margem de erro pequena.

Figura 2. Quadrirrotor desenvolvido pelo Bresciani.

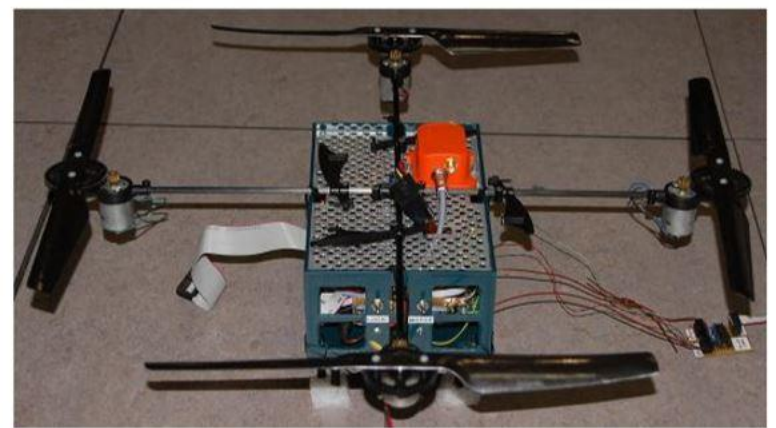

Fonte: (BRESCIANI, 2008).

Foi desenvolvido por Santana e Braga (2008) um quadrirrotor (Figura 3) na Universidade de Brasília. O autor utilizou tubos de carbono para a construção da estrutura. Como controle, o autor utilizou um microcontrolador ARM AT91. Foi utilizado acelerômetro, giroscópio e magnetômetro para compor os sensores inerciais. Em seu trabalho apresentado não teve demonstrações de testes práticos, apenas simulações.

Figura 3. Quadrirrotor desenvolvido por Santana e Braga.

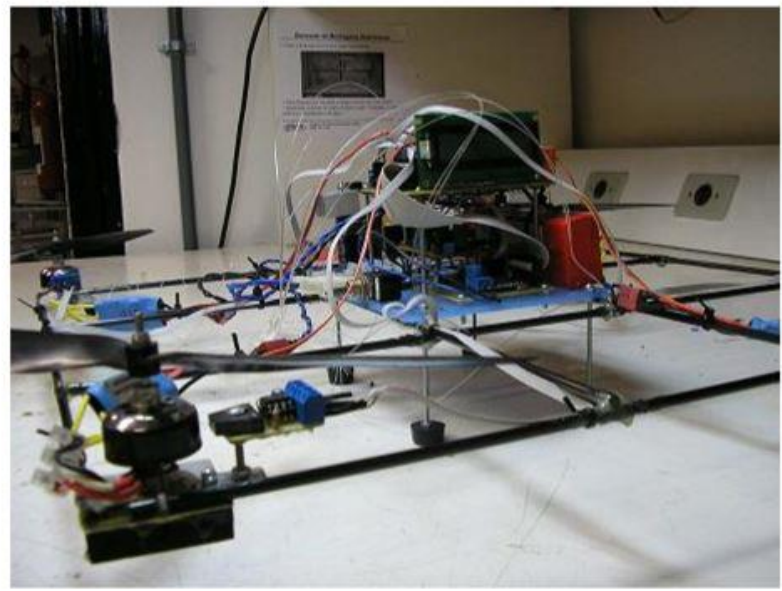

Fonte: (SANTANA; BRAGA, 2008). 
No trabalho apresentado por Paula (2012) foi desenvolvido um quadrirrotor (Figura 4), com uma estrutura constituída de tubos de fibra de carbono para os braços e para o suporte central foi utilizado o material FR41. Os sensores inerciais foram compostos de acelerômetro, giroscópio, GPS e sonar. Como controle foi utilizado o microcontrolador NXP LPC1768 fabricado pela Philips. $O$ quadrirrotor foi destinado à captura de imagens e vídeos aéreos em alta definição com custo relativamente baixo. 0 autor conclui que apesar do protótipo ser funcional, em ensaios práticos não se conseguiu obter uma estabilização mínima necessária para realizar voos.

Figura 4. Quadrirrotor desenvolvido por Paula.

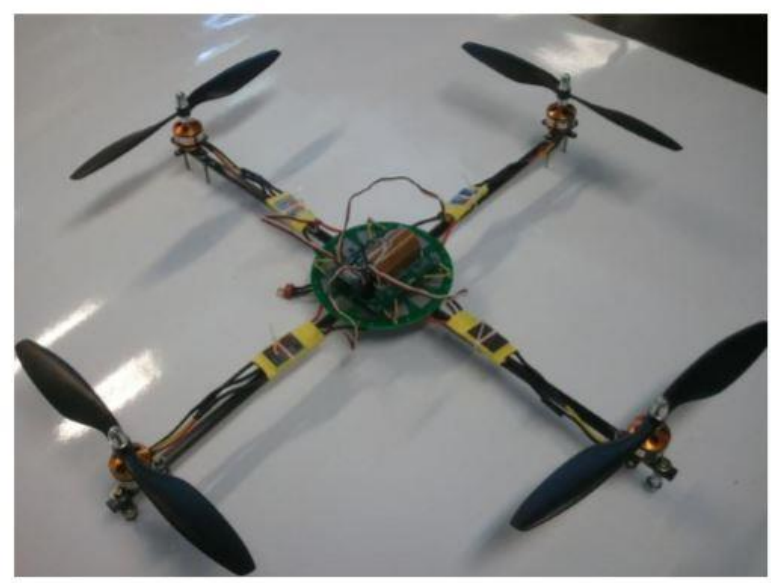

Fonte: (PAULA, 2012).

\section{METODOLOGIA PROPOSTA}

A primeira etapa do projeto foi a construção do VANT, bem como realizar a integração todas as tecnologias (sensores GPS, barômetro, magnetômetro, e XBee para comunicação) para se realizar voos controlados, com telemetria, seguindo uma rota estipulada (plano de voo).

Foi escolhido o modelo de quadrirrotor para a construção do VANT idealizado neste trabalho, que é uma espécie de helicóptero que possui uma estrutura em forma de " $X$ ", constituído de 4 motores acoplados com hélices em cada umas das extremidades. A Figura 5 mostra um esboço da estrutura base do quadrirrotor, com frente indicada pela seta, e as indicações de rotações de cada motor. Os motores representados pela cor vermelha são no sentido horário e os motores na cor azul no sentido anti-horário.

Figura 5. Esboço da estrutura do quadrirrotor (VANT).

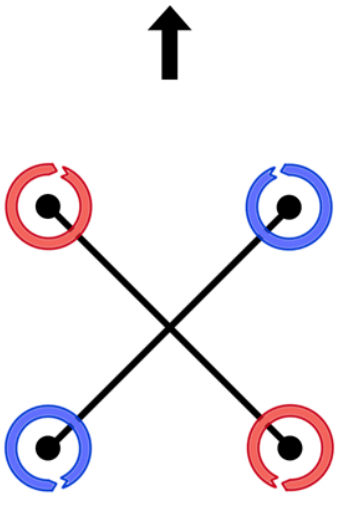

Para a construção do quadrirrotor (VANT) foram utilizados os seguintes materiais:

- 1 frame de 4 extremidades do modelo F450;

- 4 motores brushless modelo 2212 de 1000 kv;

- 2 pares de hélices de nylon $25.4 \times 11.43$ sentido horário;

- 2 pares de hélices de nylon 25.4x11.43 sentido anti-horário;

- 4 ESC (Eletronic Speed Control) controlador de velocidade dos motores;

- 1 trem de pouso;

- 2 pares de XBee PRO 60mW Wire antenna - series 1 (alcance de 1600 metros);

- 1 9-Degrees of Freedom - Sensor Stick (acelerômetro + giroscópio + Magnetômetro);

- 1 GPS shield ${ }^{2}$ with SD Slot for Arduino V2-B;

- 1 Bateria Venom LiPO 35C 3S 5500mAh 11.1V.

- 1 KK-Board. 
Para que o VANT, idealizado neste trabalho, realize voos de forma autônoma, foi desenvolvida uma controladora embarcada utilizando um Arduino Uno, que é uma plataforma de hardware Open Source contendo um microcontrolador ATmega 168/328, 14 portas digitais de entrada/saída, 6 portas analógicas de entrada, e portas seriais TX e RX. Essa controladora é responsável pelo controle de voo (para seguir a rota planejada), que para isso faz a leitura dos dados providos pelos sensores inerciais (GPS, acelerômetro, INS) embarcados no VANT, e o envio dessas informações para o acompanhamento na estação de controle (computador) via ZigBee. No software da controladora (Arduino) embarcada no VANT será utilizada a linguagem do próprio Arduino.

Quando foi criado, o padrão ZigBee teve como principal alvo aplicações de baixo consumo de energia e baixas transferências de dados (FARAHANI, 2008). O uso do ZigBee vai possibilitar receber os dados de telemetria pela estação de controle (computador em solo), até uma distância de 1600 metros (limite de alcance do módulo XBee PRO 60mW utilizado), mesmo sem um contato visual, para que o operador do sistema possa acompanhar o trajeto do VANT em um mapa na estação de controle. Caso haja necessidade de se assumir o controle manual do VANT, este poderá ser realizado pela estação de controle (computador).

A estação de controle (computador), é constituída por um computador equipado com o ZigBee e o software de controle que foi desenvolvido neste trabalho. No software foi utilizado a API do GMap.NET - Great Maps for Windows Forms \& Presentation, onde é possível gerar um plano de voo (rota), assim como acompanhar, em tempo real, a trajetória do VANT enquanto executa um voo planejado. O software da estação de controle (computador) foi desenvolvido usando o ambiente C\# (C Sharp) da plataforma .NET da Microsoft, utilizando a API do GMap (GMap.NET, 2006).
Na Figura 6 é mostrada a imagem de um Arduino Uno utilizado neste trabalho. A denominação Uno é uma nomenclatura dada à placa. Podem ser encontradas outras placas de Arduino com menor capacidade de processamento e menos portas de Entrada/Saída como, por exemplo, o Arduino Nano, e também outras placas com maiores capacidades como, por exemplo, o Arduino Mega2560. O modelo ideal escolhido para este trabalho, o Arduino Uno, possui 14 portas digitais, 6 portas PWM (Pulse Width Modulation), pode-se usar estas portas como de saída analógica, 6 portas de entrada analógicas, memória de 32 KBytes, e $16 \mathrm{Mhz}$ de poder de processamento.

Figura 6. Arduino Uno utilizado neste trabalho.

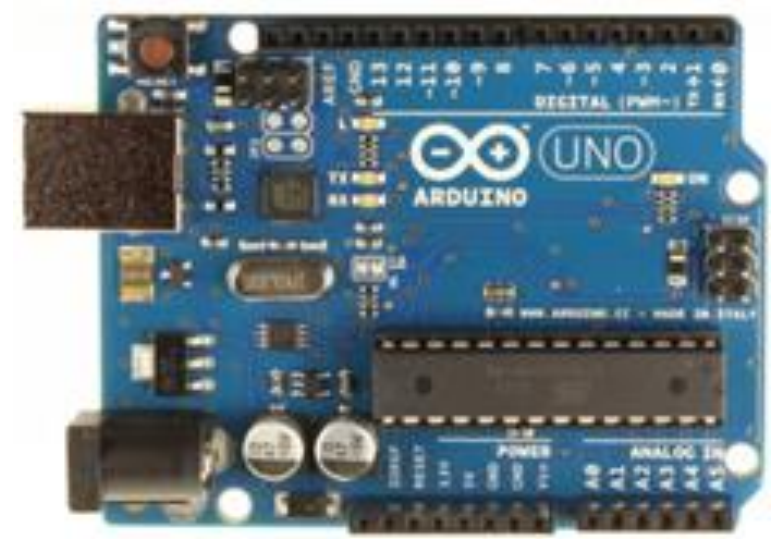

O shield de X-Bee utilizado para a comunicação entre o VANT e a Estação de controle é mostrado na Figura 7.

Figura 7. Shield X-Bee.

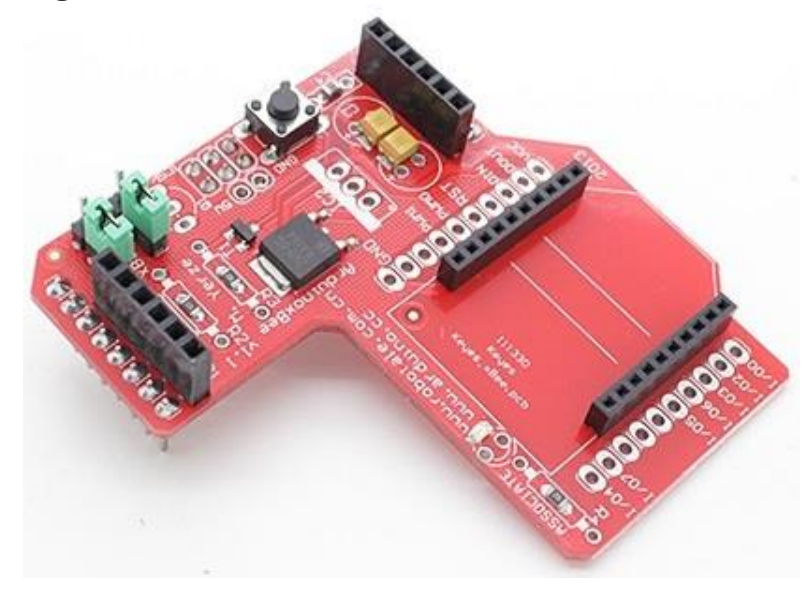


Para se obter a localização do VANT em seu trajeto, foi necessário utilizar um GPS, conforme ilustrado na Figura 8.

Figura 8. GPS.

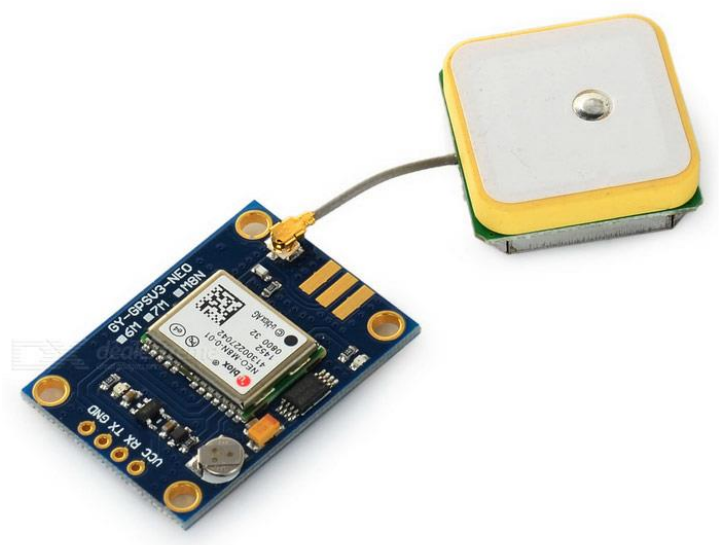

Para o controle de estabilidade do VANT, foi utilizado uma placa $K K$ Board que é composta por giroscópio, acelerômetro controlados por um Atmega324PA (Figura 9).

Figura 9. KK Board.

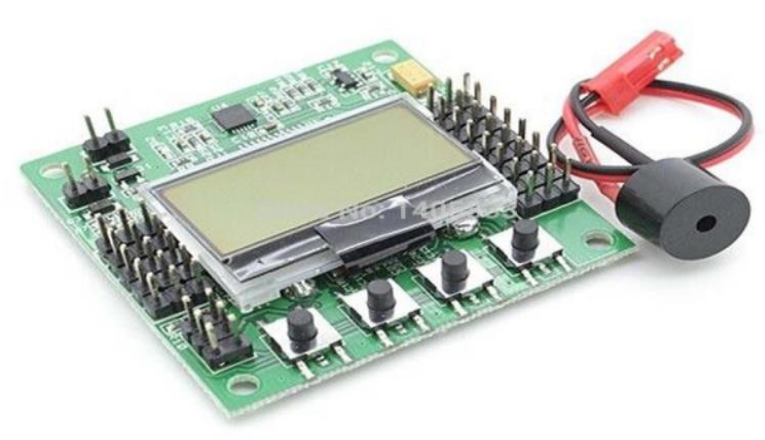

Na Figura 10 é mostrado o esquema de ligação entre o Arduino e a KK Board. A placa $K K$ Board foi utilizada para realizar a estabilidade do VANT, uma vez que ela possui acelerômetro e giroscópio, e já provê esse recurso.
Figura 10. Esquema de ligação entre o arduino e a KK Board.

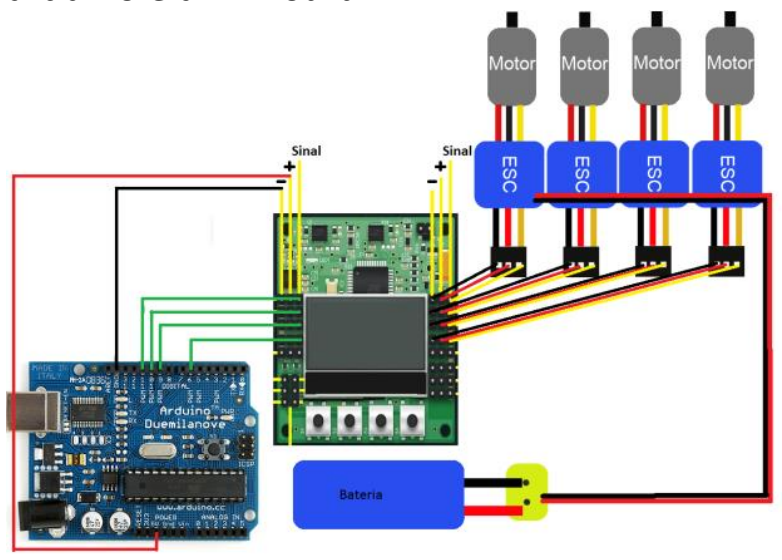

A Figura 11 mostra o esquema de ligação dos sensores (GPS, barômetro e magnetômetro) como o Arduino, bem como do shield XBee.

Figura 11. Arduino montado com os sensores de GPS (a), barômetro (b) e magnetômetro (c), e com o shield XBee (d).

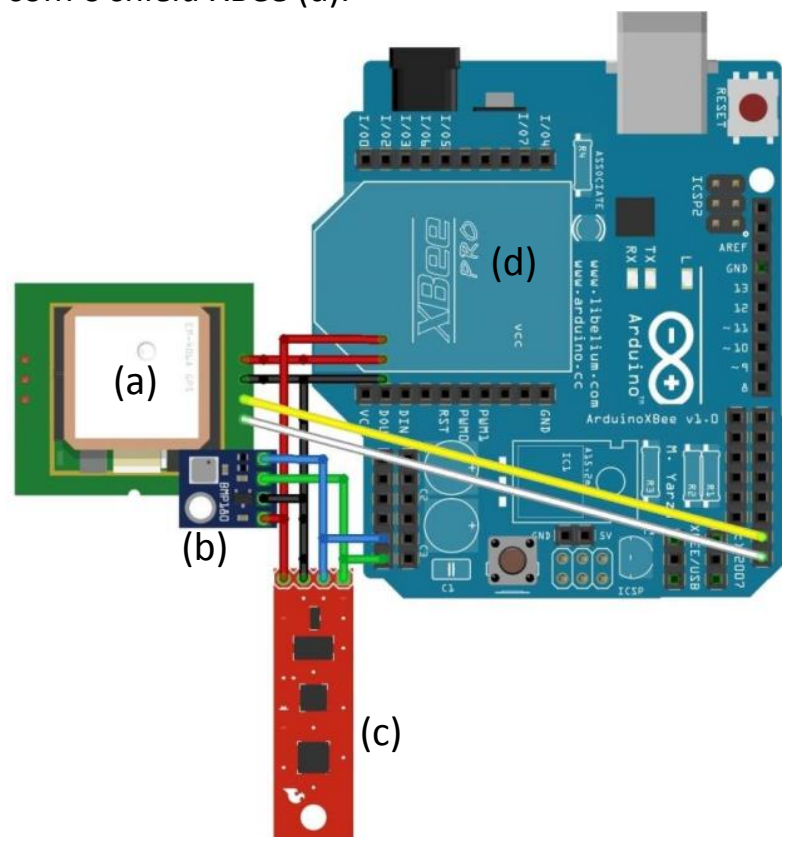

O Sistema (controladora embarcada e estação de controle em solo) montado torna possível as seguintes funcionalidades:

- Envio e recebimento de dados entre o VANT e a estação de controle;

- Captura dos dados de telemetria, latitude, longitude, altitude, velocidade, norte magnético; 
A Figura 12 apresenta o VANT montado com todos os sensores e com a controladora embarcada.

Figura 12. VANT construído neste trabalho.

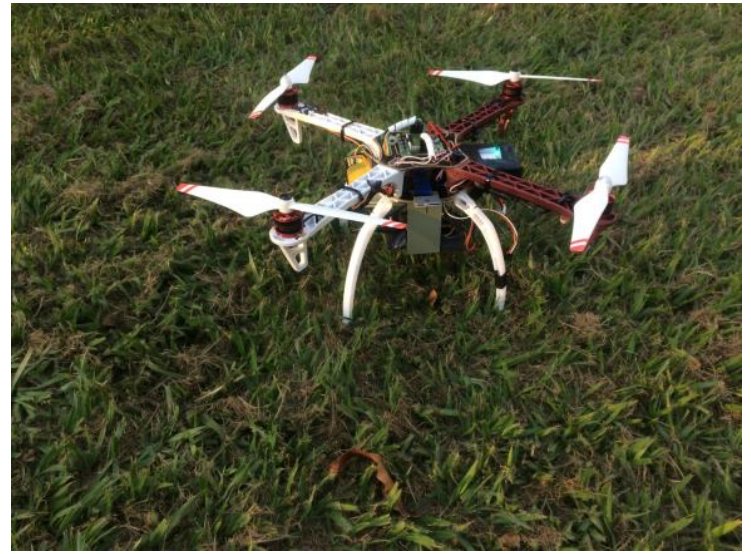

\section{EXPERIMENTOS E RESULTADOS}

Inicialmente, foram realizados testes controlados por rádio controle de 2,4 Ghz para encontrar a configuração ideal de estabilidade com a placa $K K$ Board (Figura 13). Após a configuração inicial, foram realizados voos controlados manualmente utilizando a estação de controle, porém, voos não autônomos.
Figura 13. VANT em voo por rádio controle

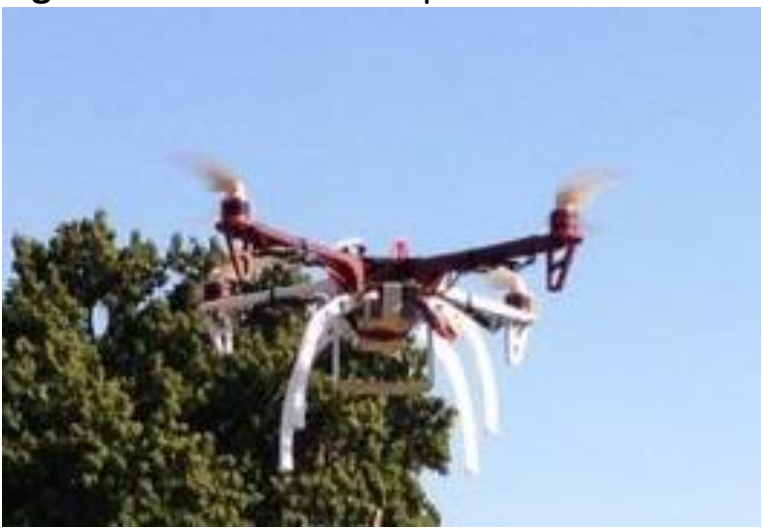

Para que o quadrirrotor pudesse percorrer uma rota de forma autônoma, foi necessário investigar uma forma de fornecer a direção e a distância correta. Chegou-se a conclusão de que encontrar o angulo da direção a ser percorrida utilizando o norte magnético (obtido pelo sensor magnetômetro) seria a melhor estratégia.

Foram realizados testes terrestres com a controladora para verificar a precisão dos dados obtidos dos sensores. Com base em uma rota criada na estação de controle (Figura 14), o trajeto foi realizado a pé, caminhando com o VANT sem as hélices, nos pontos estabelecidos para verificar se os dados coincidem com o planejado.

Figura 14. Tela da estação de controle, com os dados obtidos no teste realizado a pé com o VANT montado com a controladora embarcada.

(a)

(b)

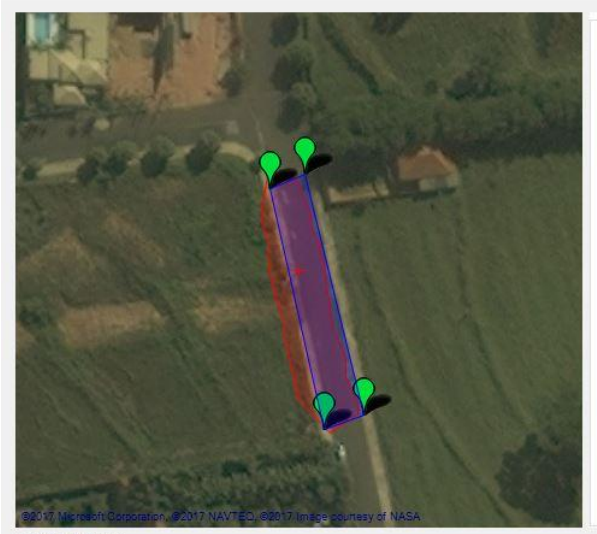
Controle do Vant
Pontos Perconidos

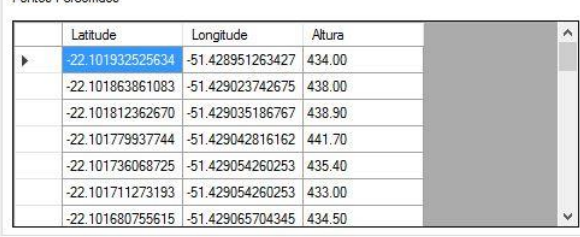

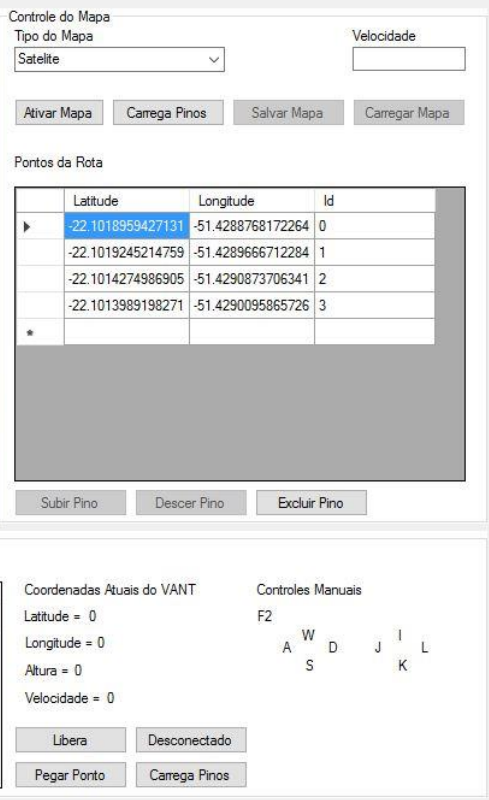

(c) 
A Figura 14 mostra a tela da estação controle, resultante do teste realizado, em que se pode observar o mapa com a rota criada (a), os pontos coletados percorridos (Latitude, Longitude e Altura) em (b), e os pontos da rota estipulada (c).

Notou-se uma eficácia não muito precisa em trajetos longos, para resolver este problema, o trajeto foi dividido em sub trechos como pontos de ajustamento de rota. A ideia é ir corrigindo parcialmente os desvios de percurso durante o voo do VANT em longas distâncias.

Para o cálculo dos sub trechos, as coordenadas de latitude e longitude foram convertidas para UTM $^{3}$ de forma a facilitar os cálculos, que após calcular os sub trechos, foram convertidos novamente para Latitude e Longitude. Quando há um erro no percurso, ele pode ser corrigido sem muitas perdas. Para os cálculos das coordenadas dos sub trechos, é utilizada a Equação (1).

$$
\begin{aligned}
E & =E+\sin (\text { angulo }) * \text { distancia } \\
N & =N+\cos (\text { angulo }) * \text { distancia }
\end{aligned}
$$

onde $E$ é a coordenada $x$ (East) e $N$ é a coordenada y (North).

A Figura 15 mostra os subtrechos da rota criada na estação de controle apresentado na Figura 14.

Figura 15. Sub trechos de uma rota criada na estação de controle.

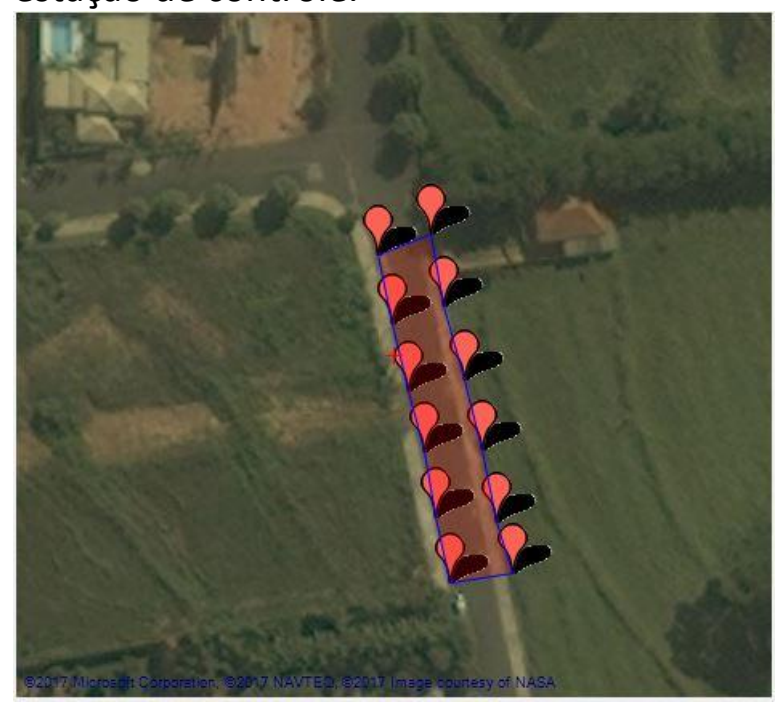

${ }^{3}$ UTM: Universal Transversa de Mercator (E, N), utiliza um sistema de coordenadas cartesianas bidimensional para dar localizações na superfície da Terra (AGUIAR; CAMARGO; GALO, 2002).

\section{CONSIDERAÇÕES FINAIS}

O objetivo deste trabalho foi desenvolver um protótipo quadrirrotor de baixo custo para realizar voos controlados de forma autônoma seguindo uma rota préestabelecida.

Foram realizados testes a pé caminhando com o VANT para verificar o funcionamento dos sensores (GPS, barômetro e magnetômetro). Testes com voos controlados, tanto com o uso de rádio controle, quanto controlados manualmente pela estação de controle, sem ligar os sensores. Estes testes serviram para testar o funcionamento da telemetria (utilizando o XBee) e da resposta do VANT em voo.

Após esta primeira etapa de testes, iniciou-se os testes com todos os sensores embarcados no VANT e ligados, porém o microcontrolador Atmel presente na placa de prototipagem Arduino não foi suficiente para tratar o gerenciamento de todos os sensores e realizar a comunicação com a estação de controle (em solo), bem como também se comunicar com a placa $K K$ Board utilizada para estabilização. Isso fez com que os testes de voo autônomo fossem abortados. Assim como em Bresciani (2008), Santana e Braga (2008) e Paula (2012), a solução encontrada, porém não implementada neste trabalho, seria utilizar um microprocessador $\mathrm{ARM}^{4}$ para construir a controladora do VANT. Para simplificar essa solução proposta para trabalhos futuros, poderia ser utilizado uma placa Raspberry $\mathrm{Pi}$, que possui um microprocessador de 32 bits com portas de comunicação semelhantes as encontradas no Arduino.

\section{REFERÊNCIAS}

AGUIAR, C. R.; CAMARGO, P. O.; GALO, M. Transformação de Coordenadas e Datum com Propagação de Covariâncias. In: SIMPÓSIO BRASILEIRO DE GEOMÁTICA, Anais... Presidente Prudente - SP, 9-13 de julho de 2002. p.113-120.

\footnotetext{
${ }^{4}$ Arquitetura ARM (Advanced RISC Machine) é uma arquitetura de processador de 32 bits usada principalmente em sistemas embarcados.
} 
ARDUINO. 2015. Disponível em: <https://www.arduino.cc/en/Main/ArduinoS hields>. Acesso em: 10 jul. 2015.

ARMADA. The Growing World of Unmanned Airborne Spies. Armada International, v. 28, n. 3, 2004.

BRESCIANI, T. Modelling, Identification and Control of a Quadrotor Helicopter. Lund, 2008. 184p. Tese (Master Thesis) - Lund University, Suécia, 2008.

EISENBEI, H. UAV Photogrammetry. Dresden, 2009. 235p. Dissertação de mestrado University of Technology Dresden, Alemanha, 2009.

FARAHANI, S. ZigBee Wireless Network and transceivers. Burlington: Newnes, 2008.

FOWERS, S. G. Stabilization and control of a quad-rotor micro-uav using vision sensors. Provo, 2008. 112p. Dissertation (Master of Science) - Brigham Young University, Estados Unidos, 2008.

GMap.NET. Great Maps for Windows Forms \& Presentation. 2006. Disponível em: $<$ https://greatmaps.codeplex.com/>. Acesso em: 25 fev. 2016.

LEISHMAN, J. G. The Breguet-Richet Quadrotor Helicopter of 1907. Vertiflite, v. 47, n. 3, p. 58-60, 2002.

LONGHITANO, G. A. Vants para sensoriamento remoto: Aplicabilidade na avaliação e monitoramento de impactos ambientais causados por acidentes com cargas perigosas. São Paulo, 2010. 148p. Dissertação (Mestrado) - Escola Politécnica da Universidade de São Paulo, Departamento de Engenharia de Transportes, 2010.

PAULA, J. C. Desenvolvimento de um VANT do tipo Quadrirrotor para obtenção de imagens aéreas em alta definição. Curitiba $P R$, 2012. 104p. Dissertação (Mestrado em
Engenharia Elétrica) - Programa de PósGraduação em Engenharia Elétrica, Setor de Tecnologia, Universidade Federal do Paraná UFPR, 2012.

SANTANA, P. H. R. Q.; BRAGA, A. S. M. A. Concepção de um Veículo Aéreo NãoTripulado do Tipo Quadrirrotor. Brasília, 2008. 164p. Monografia (Trabalho de Graduação) - Universidade de Brasília, Brasília, 2008.

US DEPARTMENT OF DEFENSE. Dictionary of Military and Associated Terms. 2010. Disponível em: $<$ http://www.dtic.mil/doctrine/ new_pubs/jp1_02.pdf>. Acesso em: 25 fev. 2016.

WONG, K.C. et al. Study of the Unmanned Aerial Vehicle (UAV) Market in Australia. Aerospace Technology Forum Report, 1997. 